Article

\title{
A Target-Temperature Monitoring System for Cyclotron Targets: Safety Device and Tool to Experimentally Validate Targetry Studies
}

\author{
Sergio J. C. do Carmo ${ }^{1, *(\mathbb{B})}$, Pedro M. de Oliveira ${ }^{1}$ and Francisco Alves ${ }^{2,3}$ (1) \\ 1 ICNAS-Produção, University of Coimbra, Pólo das Ciências da Saúde, Azinhaga de Santa Comba, \\ 3000-548 Coimbra, Portugal; p.de.oliveira20@gmail.com \\ 2 Institute for Nuclear Sciences Applied to Health (ICNAS), University of Coimbra, \\ Pólo das Ciências da Saúde, Azinhaga de Santa Comba, 3000-548 Coimbra, Portugal; franciscoalves@uc.pt \\ 3 Instituto Politécnico de Coimbra (IPC), Coimbra Health School, 3046-854 Coimbra, Portugal \\ * Correspondence: sergiocarmo@uc.pt; Tel.: +351-239-488-510
}

Received: 17 May 2018; Accepted: 19 June 2018; Published: 21 June 2018

\begin{abstract}
The present work describes an experimental system enabling temperature measurement in cyclotron targets' cooling water during bombardment. The developed system provides sensible and immediate response to variations of irradiation conditions during bombardment and enables quantification of the temperature rise in the cooling water due to beam interaction with the irradiated target and with its collimator. Such a system finds application either as a monitoring safety device to instantaneously detect and register abnormal alterations in target conditions to anticipate thermal-related incidents and as a tool to experimentally validate cyclotron targetry optimization studies and thermal simulations.
\end{abstract}

Keywords: cyclotron; targetry; thermal study

\section{Introduction}

As the production yield of radionuclides in cyclotrons depends on the interaction of the beam with the target material, a large amount of studies have been conducted over the last decades to study beam/target interfaces [1-3], regardless of gas, liquid, or solid phase of target material. These studies range from beam characterization and target design to thermal modelling; commonly aiming at improving and optimizing the production processes. A shared concern consists of maintaining the target temperature relatively low despite the considerable temperature rise generated by the beam interaction to maximize the possible target current and in order to guarantee that the impact of the impinging particles have no repercussion on the radionuclide production process and/or damage the target. For instance, several authors reported distinct problems arising from the very high temperature increment within the target during irradiation, such as melting or even evaporation of the target material in solid targets (in most cases, cost-prohibitive enriched material), sometimes resulting in a degradation of high-vacuum or damage on the backing target components [4]. There is therefore an interest in monitoring the target temperature for safety reasons, to anticipate and avoid temperature-related incidents in order to obtain the desirable production yields. However, while temperature monitoring is well established for the solid target technique, little empirical information is available concerning liquid or gas targets [5,6]. Besides, since combined thermal and fluid simulations are widely used in designing and optimizing cyclotron targets, experimental temperature monitoring devices are also of great importance in assessing target performance and to experimentally validate the results of thermal simulation studies [7]. 
The present work describes an experimental system used to measure the temperature of the water used to cool liquid targets during irradiation when it thermally interacts with the irradiated liquid-target arrangement. The developed system is not only sensitive to beam variations but it also quantifies the temperature increments due to beam interaction within the target, so it can be used either for safety monitoring applications or as a tool to experimentally validate results from thermal simulation studies.

\section{Materials and Methods}

Three thermistors were implemented in the cooling system arrangement of a commercial IBA conical-shaped Nirta $\mathrm{C} 8^{\circledR}$ niobium liquid target [8,9]; adapted to measure the temperature of the cooling water at the back of the niobium insert, i.e., the inlet temperature, and at the exits of the target and collimator. Two thermistors (screw type pipe probes from Semitec Corporation (Tokyo, Japan) [10]) were placed at the exits of the target and collimator internal water channels. The third transducer was installed at the back of the niobium insert, also placed inside the water cooling tunnel, and was custom-made to fit inside the existing geometry without interfering the water flow and so the target performance. This is a $1.5 \mathrm{~mm}$ diameter and $100 \mathrm{~mm}$ long rod manufactured by USSensor Corporation (Orange, CA, USA) [11], thermally insulated up its extremity since it is totally inserted in the cooling channel. The distance separating the back of the conical shaped target and the thermistor, typically $1 \mathrm{~mm}$, can be adjusted and minimized down to contact by screwing the back end of the thermistor to the adapted diffuser of the refrigerated water. As a result, cooling water temperature can be measured exactly before interacting with the back of the niobium target insert and immediately after exiting the target and the collimator, thus making it possible to quantify the temperature increments in the cooling water due to the beam interaction in both the target and the collimator separately. The thermistors placed within the liquid target setup were connected outside the cyclotron vault to voltage dividers in order to determine their temperature-dependent resistance during irradiation. The measured voltages were converted to temperature measurements using an Arduino-based interface [12] registering the data thanks to a PC-based data logger terminal [13].

\section{Results}

Figure 1 presents the temperature of the refrigerated water at the back of the target insert, at the exit of the target and at the exit of the collimator arrangements as a function of time in a typical irradiation of enriched ${ }^{18} \mathrm{O}$-water for the production of ${ }^{18} \mathrm{~F}$. It also shows quantification of the rise in temperature from the target and the collimator during bombardment. Figure 1 confirms that the three measured temperatures are identical whenever there is no beam on the target and that the temperature of the inlet cooled water at the back of the target insert is as expected always similar to the temperature of the refrigerated water supplied to the cyclotron vault. Figure 1 also shows that although the temperature of the refrigerated water only stabilizes after a long period (due to slow thermal stabilization in the different vast sub-systems cyclotron; such as coils of the magnetic field, radiofrequency structure, etc.), rising temperature occurs and stabilizes instantaneously in the target and the collimator, as already reported by Steinbach et al. [14]. Moreover, Figure 1 indicates that the temperature increments seem to be almost independent of the refrigerated water temperature since these are nearly constant during the irradiation.

In order to quantify the increment in temperature of the cooling water due to the target + collimator arrangement only, overall thermal stabilization of the cyclotron was reached before irradiating the target. The referred temperatures were registered as the several distinct sub-systems of cyclotron were switched on individually; waiting each time for thermal stabilization before switching on the next sub-system. As can be seen in Figure 2, the heat load due to target irradiation is not macroscopically relevant when compared to the overall heat exchange from the accelerator since it does not result in an additional temperature increment in the cooling water. 


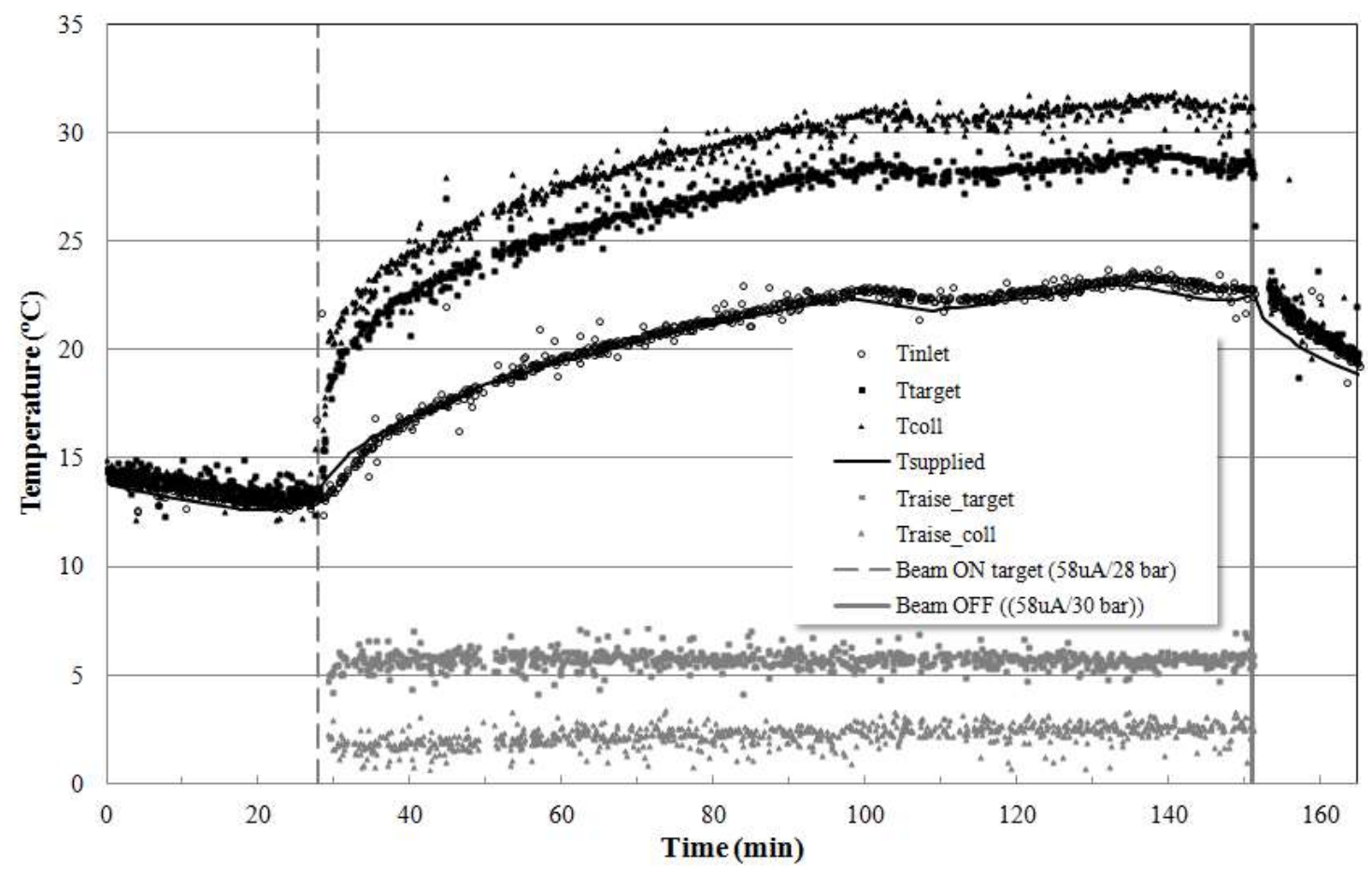

Figure 1. Temperature of the refrigerated water (black symbols) at the back of the target insert (Tinlet), at the exit of the target (Ttarget), and at the exit of the collimator (Tcoll) and temperature of the supplied cooling water (Tsupplied) as a function of time. The resulting temperature increases (grey symbols) inside the target and the collimator are also represented.

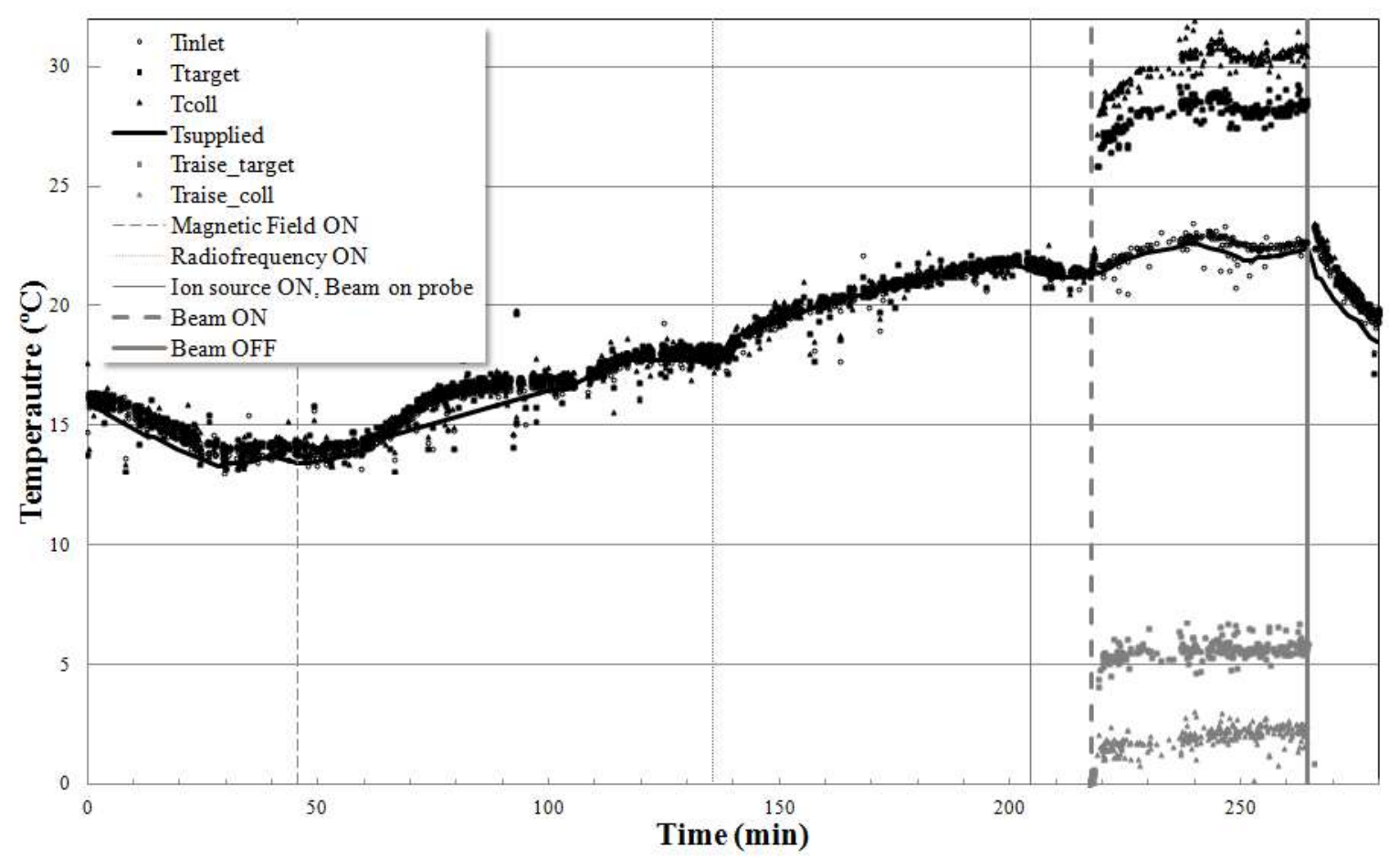

Figure 2. Temperature of the refrigerated water (black symbols) at the back of the target insert (Tinlet), at the exit of the target (Ttarget), at the exit of the collimator (Tcoll) and temperature increases inside the target and the collimator (grey symbols) as a function of time when each cyclotron thermally-relevant sub-system is switched on one by one after thermal stabilization is reached. 
The temperature increments as a function of the beam current on target were also investigated. For that purpose, thermal stabilization of the cyclotron was previously reached and maintained by irradiating another identical target long enough to reach thermal equilibrium. Figure 3 confirms that thermal equilibrium was previously reached as the measured temperatures are stable and the only variations registered were due to, and follow, cyclic variations in the temperature of the cooling water in the primary cooling circuit, arising from operating cycles of the chiller unit. Only then was the target of interest irradiated, with no resulting global thermal change. Figure 3 shows that the temperature increments in the target and the collimator depend on the beam current and that the developed system is sensitive to such thermal changes, while the global system registers no macroscopic temperature change since thermal equilibrium was previously guaranteed. The technique is sensitive to such temperature changes and registers these alterations and allows their quantification; as illustrated in Figure 4 where the temperature increases in both the target and the collimator are represented for distinct beam currents. Such beam current dependence was expected since, at thermal equilibrium (i.e., time-independent), Newton's law of cooling shows that the rate of convection heat transfer and the difference in temperatures between the liquid target and its surroundings are proportionally related through the equation

$$
\frac{d Q}{d t}=I_{\text {target }} \Delta E=h A\left(T_{\text {target }}-T_{w}\right)
$$

where $Q$ is the thermal energy (in J), $I_{\text {target }}$ is the target beam current, $\Delta E$ is the energy loss within the liquid target (i.e., $18 \mathrm{MeV}), h$ is the convective heat-transfer coefficient (in $\mathrm{W} /\left(\mathrm{m}^{2} \cdot \mathrm{K}\right.$ ) and assumed to be independent of the temperature), $A$ is the heat transfer surface (i.e., the inner surface of the niobium cavity (in $\left.\left.\mathrm{m}^{2}\right)\right), T_{\text {target }}$ is the temperature of the liquid target water (assumed to be constant), and $T_{w}$ is the temperature of the refrigerated water. Figure 5 illustrates and confirms the expected proportion between the temperature increments in the cooling water and the beam current.

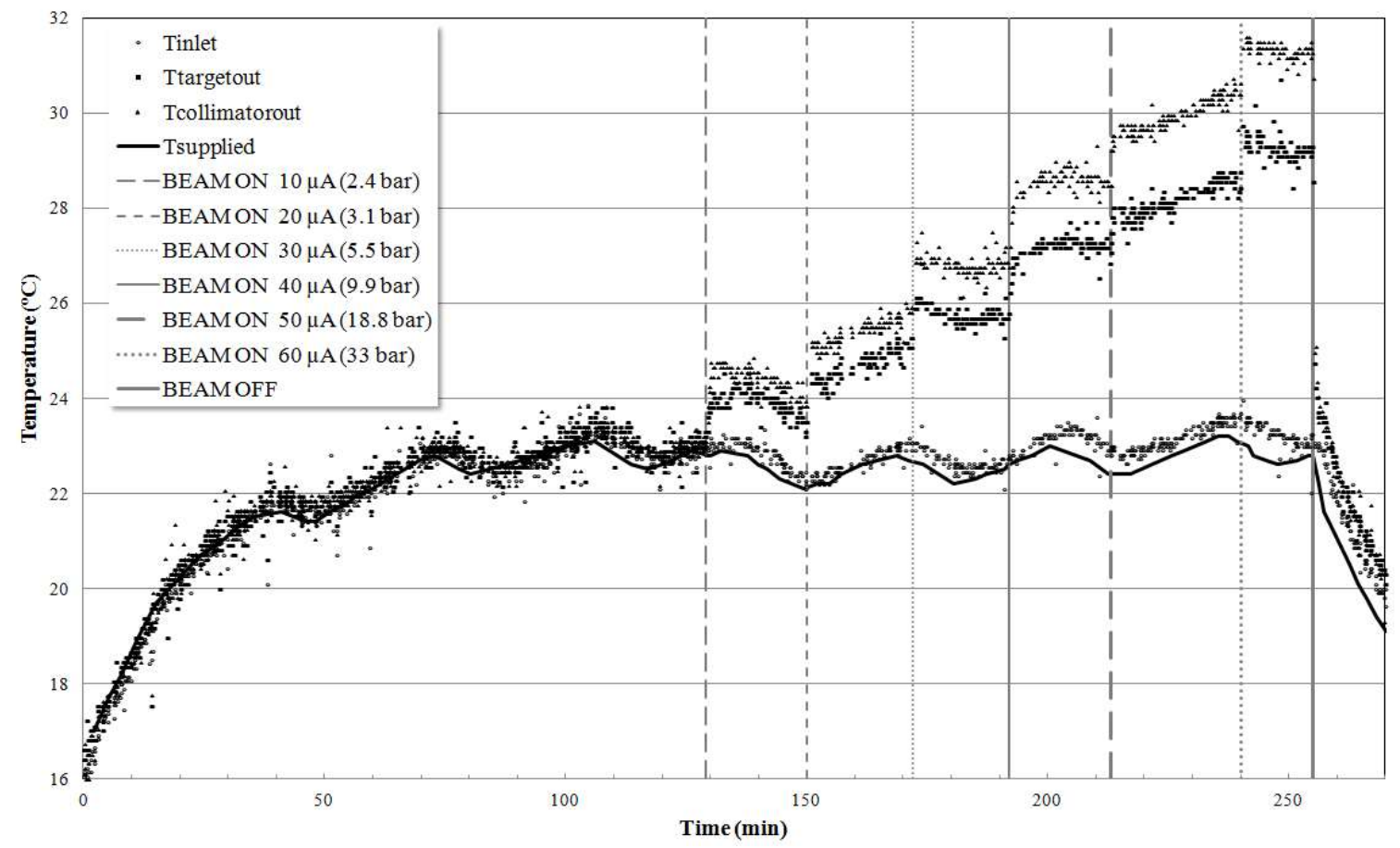

Figure 3. Temperature of the refrigerated water at the back of the target insert (Tinlet), exits of the target (Ttargetout), and exit of the collimator (Tcollimatorout) as a function of time after thermal stabilization and for distinct beam currents on the target. 


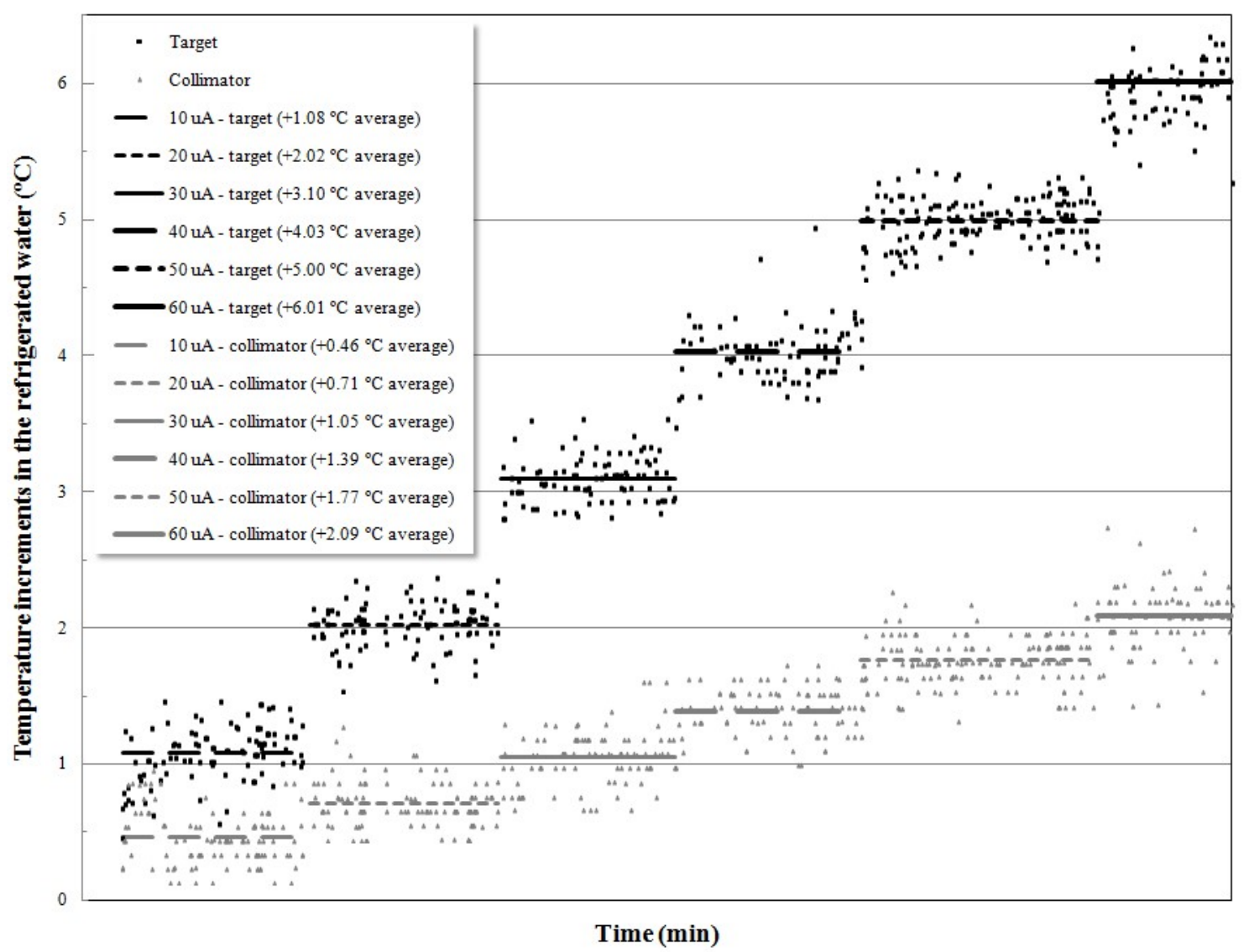

Figure 4. Temperature increases in the target and the collimator with respect to the temperature of the refrigerated water at the back of the target insert (Tinlet) as a function of time after thermal stabilization and for distinct beam currents on the target.

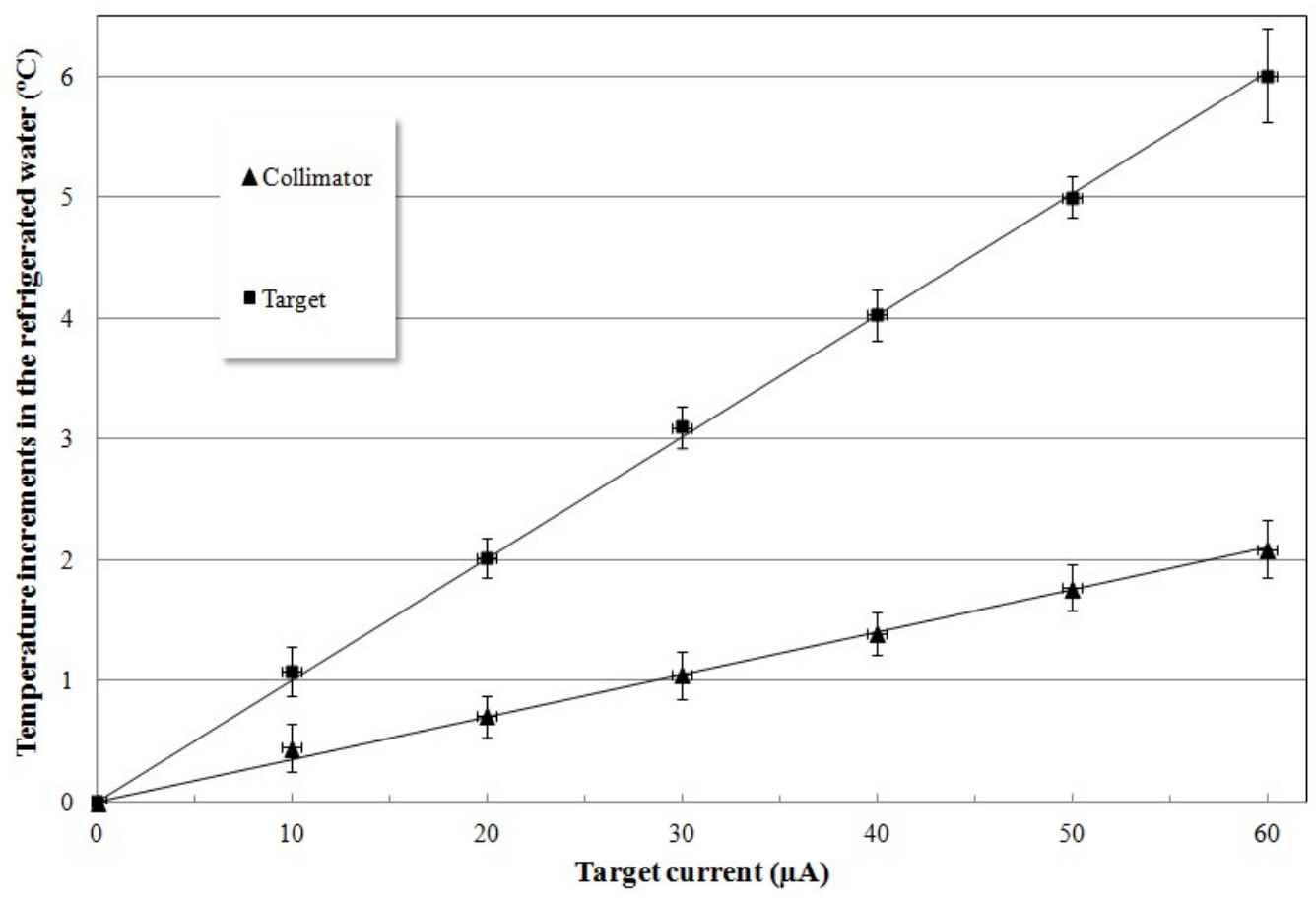

Figure 5. Temperature increases in the target and the collimator as a function of the beam current on target. 
As stated by Equation (1), the heat transfer depends on the temperature differential; i.e., on the temperature of the refrigerated cooling water. As a result, there was also interest in registering the evolution of the referred temperatures of interest over a typical irradiation, during which the temperature of the cooling water was altered. For that purpose, the technique previously described was adopted, i.e., another identical target was irradiated long enough to reach thermal equilibrium. Figure 6 shows that even if each individual measured temperature is particularly dependent of the temperature of the refrigerated water, the temperatures increments are almost identical for three broadly distinct water cooling temperatures. The temperature increments indeed increase as the temperature of the cooling water decreases (i.e., as the temperature differential increases, as expected from Equation (1)) but Figure 6 also shows that these variations are marginal. This result confirms that heat exchange is mostly dependent on the liquid-target surface available and on its capacity to effectively remove the heat generated in the liquid from the beam interaction, as pointed out by Steyn et al. [6].

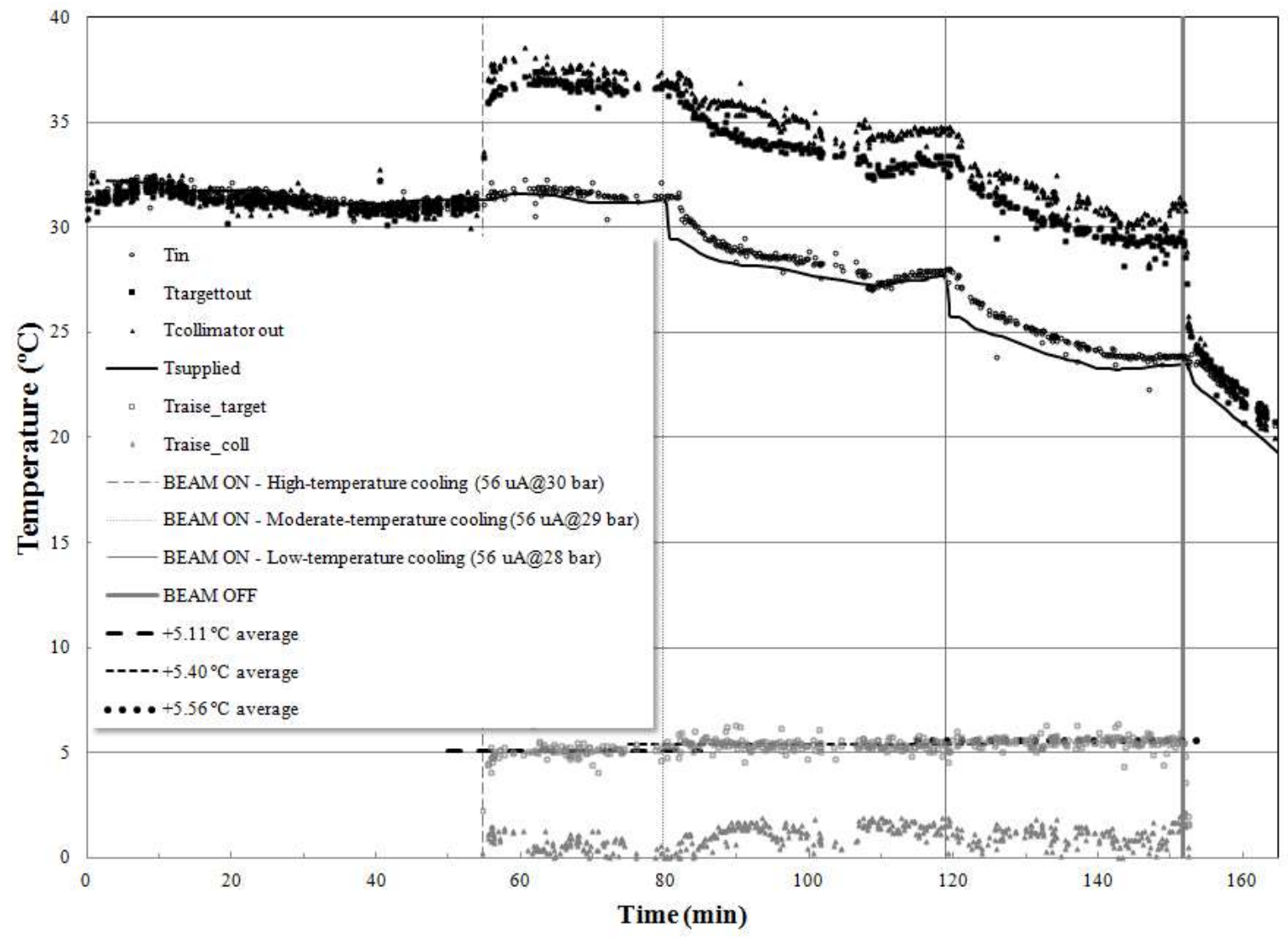

Figure 6. Temperatures of the refrigerated water (black symbols) at the back of the target insert (Tin), at the exits of the target (Ttargetout), at the exit of the collimator (Tcollimatorout) and temperature increases inside the target and the collimator (grey symbols) as a function of time after thermal stabilization and with the supplied refrigerated water at 3 distinct temperatures during irradiation.

\section{Conclusions}

The present work describes an experimental set-up used to measure the temperature of the cooling water in a liquid or gas target under irradiation. The system is sensitive to variations of the conditions of irradiation during bombardment, providing an immediate and quantitative response to the rise in temperature in the refrigerated water. The measurements confirmed the relationship between the temperature increments in the cooling water and the beam current. These also demonstrate that the initial temperature of the refrigerated water is not relevant to the thermal 
exchange, and so too the target performance, since the temperature of the liquid target inside the cavity is greatly superior. This particular result confirms that the target performance depends mostly on the liquid-target surface available and its capacity to effectively remove the heat generated. The technique developed can be used to experimentally validate cyclotron-targetry optimization studies and thermal simulations. On the other hand, it is also useful as a safety interlock to instantaneously detect and register abnormal alterations in the irradiation conditions during bombardment in order to anticipate thermal-related incidents.

Author Contributions: Conceptualization, S.J.C.d.C.; Methodology, S.J.C.d.C.; Software, S.J.C.d.C.; Validation, S.J.C.d.C.; Formal Analysis, P.M.d.O.; Investigation, S.J.C.d.C.; Writing-Original Draft Preparation, S.J.C.d.C.; Writing-Review \& Editing, F.A.; Visualization, S.J.C.d.C.; Supervision, F.A.; Project Administration, F.A.

Funding: This research received no external funding.

Acknowledgments: The authors want to acknowledge Daniel Schiemann from SEMITEC Corporation for generously donating thermistors used in the experiments.

Conflicts of Interest: The authors declare no conflict of interest.

\section{References}

1. Nortier, F.M.; Stevenson, N.R.; Gelbart, W.Z. Investigation of the thermal performance of solid targets for radioisotope production. Nucl. Instrum. Methods 1995, A355, 236-241. [CrossRef]

2. Heselius, S.-J.; Lindblom, P.; Solin, O. Optical studies of the influence of an intense ion beam on high-pressure gas targets. Int. J. Appl. Radiat. Isot. 1982, 33, 653-659. [CrossRef]

3. Helus, F.; Uhlir, V.; Gasper, H. Contribution to cyclotron targetry: V. Measurement of the temperature in a gas target during irradiation. J. Radioanal. Nucl. Chem. 1996, 210, 233-235. [CrossRef]

4. McCarthy, D.W.; Shefer, R.E.; Klinkowstein, R.E.; Bass, L.A.; Margeneau, W.H.; Cutler, C.S.; Anderson, C.J.; Welch, M.J. Efficient production of high specific activity $64 \mathrm{Cu}$ using a biomedical cyclotron. Nucl. Med. Biol. 1997, 24, 35-43. [CrossRef]

5. Steyn, G.F.; Vermeulen, C. A Saturation Boiling Model for an Elongated Water Target Operating at a High Pressure during ${ }^{18}$ F Production Bombardments. Journal of Physics: Conference Series 2014. Available online: http:/ / events.saip.org.za/getFile.py/access? contribId=329\&sessionId=30\&resId=1\&materialId= paper\&confld=14 (accessed on 9 May 2018).

6. Steyn, G.F.; Vermeulen, C. Saturation conditions in elongated single-cavity boiling water targets. In Proceedings of the 15th international Worshop on Targetry and Target Chemistry-WTTC, Prague, Czech Republic, 18-21 August 2014; pp. 149-150.

7. Gagnon, K.; Wilson, J.S.; Quarrie, S.A. Thermal modelling of a solid cyclotron target using finite element analysis: An experimental validation. In Proceedings of the 13th international Worshop on Targetry and Target Chemistry-WTTC, Roskilde, Denmark, 26-28 July 2010; p. 11.

8. IBA, Louvain-la-Neuve, Belgium. Available online: https://www.iba-radiopharmasolutions.com/ (accessed on 20 June 2018).

9. Devillet, F.; Geets, J.-M.; Ghyoot, M.; Kral, E.; Natergal, B.; Mooij, R.; Vosjan, M. Performance of IBA new conical shaped niobium $\left[{ }^{18} \mathrm{O}\right]$ water targets. In Proceedings of the 15th international Worshop on Targetry and Target Chemistry-WTTC, Prague, Czech Republic, 18-21 August 2014; pp. 145-148.

10. SEMITEC Corporation, Japan. Available online: http:/ / www.semitec.co.jp/ (accessed on 20 June 2018).

11. USSENSOR CORP., USA. Available online: http:/ / www.ussensor.com/ (accessed on 20 June 2018).

12. Arduino. Available online: http://www.arduino.cc/ (accessed on 20 June 2018).

13. CoolTermWin Software Package. Available online: http:/ / freeware.the-meiers.org/ (accessed on 20 June 2018).

14. Steinbach, J.; Guenther, K.; Loesel, E.; Grunwald, G.; Mikecz, P.; Ando, L.; Szelecsenyi, F.; Beyer, G.J. Temperature Course in small volume $\left[{ }^{18} \mathrm{O}\right]$ water targets for $\left[{ }^{18} \mathrm{~F}\right]$ F-production. Appl. Radiat. Isot. 1990, 41, 753-756. [CrossRef]

(C) 2018 by the authors. Licensee MDPI, Basel, Switzerland. This article is an open access article distributed under the terms and conditions of the Creative Commons Attribution (CC BY) license (http:/ / creativecommons.org/licenses/by/4.0/). 